\title{
The combined effects of genetic variation in the SIRT1 gene and dietary intake of $n-3$ and $n-6$ polyunsaturated fatty acids on serum LDL-C and HDL-C levels: a population based study
}

\author{
Tomoko Inamori ${ }^{1}$, Toshinao Goda ${ }^{2}$, Nobuhiko Kasezawa ${ }^{3}$ and Kimiko Yamakawa-Kobayashi ${ }^{{ }^{*}}$
}

\begin{abstract}
Background: Dyslipidemia due to high total cholesterol, LDL-cholesterol, triglycerides, or low HDL-cholesterol is an important risk factor for coronary heart disease (CHD). Both SIRT1 and PUFAs can influence the expression of genes for nuclear receptors and transcription factors related to lipid metabolism such as LXRa, LXRß, PPARa, SREBP-1C.

Methods: A total of 707 Japanese males and 723 females were randomly selected from the participants who visited a medical center for routine medical check-ups. We analyzed the combined effects of the genotype/ haplotype of the SIRT1 gene and dietary n-6/n-3 PUFA intake ratio on the determination of serum lipid levels.

Results: We found that the SIRT1 gene marked with haplotype 2 was associated with decreased serum LDL-cholesterol and increased HDL-cholesterol levels. In addition, the associations between the SIRT1 haplotype 2 and decreased LDL-C and increased HDL-C levels were only observed in the low n- $6 / n-3$ PUFA intake ratio group, but not in the high n-6/n-3 PUFA intake ratio group.

Conclusions: Our findings indicate that the combination of genetic variation in the SIRT1 gene and dietary n- 6 and/or n-3 PUFA intake influence the determination of inter-individual variations of serum levels of LDL-C and HDL-C.
\end{abstract}

Keywords: LDL-cholesterol, HDL-cholesterol, SIRT1, Fatty acids

\section{Background}

Dyslipidemia due to high total cholesterol, low density lipoprotein (LDL)-cholesterol, triglycerides, or low high density lipoprotein (HDL)-cholesterol is an important risk factor for coronary heart disease (CHD) [1,2]. Data from family and twin studies suggest that genetic variations account for $40-60 \%$ of the inter-individual variations in plasma lipid levels [2,3]. In addition to rare mutations that cause familial dyslipidemia, common genetic variants are considered to significantly contribute to the heritability of plasma lipid levels. For example, genome-wide association studies (GWAS) have reported a growing number of new loci involved in lipid metabolism [4,5]. However, loci identified

\footnotetext{
* Correspondence: kobayasi@u-shizuoka-ken.ac.jp

'Laboratory of Human Genetics, School of Food and Nutritional Sciences, Graduate School of Integrated Pharmaceutical and Nutritional Sciences, University of Shizuoka, Shizuoka 422-8526, Japan

Full list of author information is available at the end of the article
}

through GWAS may not fully explain the inter-individual variation in plasma lipid levels.

Sirtuin 1 (SIRT1) belongs to the sirtuin protein family of nicotinamide adenine dinucleotide $\left(\mathrm{NAD}^{+}\right)$-dependent histone deacetylases conserved in evolution from bacteria to humans [6,7]. Human have seven sirtuin family members, SIRT1-SIRT7, which exhibit with different cellular locations, enzyme activities, target substrates and tissuespecificity. Of these, SIRT1 has been most extensively studied. SIRT1 is a nuclear protein and promotes chromatin silencing and transcriptional repression through histone deacetylation. In addition, more than a dozen non-histone proteins serve as substrates for SIRT1. SIRT1 controls numerous physiological processes and protects cells against stress. A number of studies have shown that SIRT1 orthologs are important mediators of the extension of life span observed from yeast to mammals following calorie restriction. During energy crises such as calorie restriction, 
$\mathrm{NAD}^{+}$level rise, concomitant with SIRT1 activation [6,7]. Transgenic mice overexpressing SIRT1 have beneficial calorie restriction-like phenotypes, while down-regulation of SIRT1 accelerates the aging phenotype in mice [8].

Furthermore, SIRT1 also has an important function in lipid and glucose metabolism, due to deacetylation of a number of nuclear receptors and transcription factors related to lipid and glucose metabolism such as peroxisomeproliferator activated receptor (PPAR) $\alpha$, PPAR $\gamma$, peroxisome proliferator-activated receptor gamma coactivator 1- $\alpha$ (PGC1- $\alpha$ ), liver X receptor (LXR) $\alpha, \mathrm{LXR} \beta$, forkhead box $\mathrm{O}$ (FOXO), AMP-activated protein kinase (AMPK) and sterol response element-binding protein-1c (SREBP-1c) [6-11]. Thus, SIRT1 is associated with lipid metabolism, and variations of the SIRT1 gene might affect the determination of inter-individual variations of plasma lipid levels.

Fatty acids are no longer just sources of energy, but also fine modulators of cellular signaling and metabolism [12]. There is growing evidence of health benefits of consuming certain types of fats including n- 6 and n-3 polyunsaturated fatty acids (PUFAs) [12,13], which are essential fatty acids that are not synthesized de novo by mammals. Several studies have shown that dietary intake of n-6 PUFAs, such as linoleic acid found in vegetable oils, may reduce CHD risk by beneficial effects on serum total cholesterol, LDL cholesterol, and insulin sensitivity [14], while n-3 PUFA derived from fish has also been shown to decrease serum triglyceride and increase HDL-cholesterol, which is associated with more efficient reverse cholesterol transport and a reduced risk of CHD $[15,16]$. It is also reported that n-3 PUFA have anti-inflammatory effects [16]. However, the cellular mechanisms underling the beneficial effects of n- 6 and n-3 PUFA on lipid profile and CHD prevention are not completely understood. Recently, n-6 and n-3 PUFAs were shown to be critical for modulation of expression in several nuclear receptors and transcription factors, including LXR $\alpha, \mathrm{LXR} \beta$, PPAR $\alpha$, SREBP-1c, hepatocyte nuclear factor (HNF)-4 $\alpha$, and nuclear factor- $\mathrm{kB}$ (NFkB) [17]. The majority of these genes play key roles in lipid metabolism, and their expression is also modulated by SIRT1 [6-11]. Furthermore, it was reported that calorie restriction and dietary n-3 PUFA intake induce the similar beneficial effects such as anti-inflammation, preventing obesity and increasing expression insulin sensitivity in mice [18]. Recently, it was shown that the anti-inflammatory effect of n-3 PUFAs might be mediated through activation of AMPK/SIRT1 pathways; because of $n-3$ PUFAs increased expression, phosphorylation and activity of AMPK in macrophages, which further leaded to SIRT1 over-expression [19]. These data indicate the possibility that dietary n-3 PUFAs intake modify the SIRT1 activity in vivo.

In the present study, we investigated whether the common variations in the SIRT1 gene are potential contributors to inter-individual variations in serum lipid levels.
Furthermore, we analyzed the interaction of the common SIRT1 variants and dietary n-3 and n-6 PUFAs intake on determination of serum lipid levels.

\section{Results}

Table 1 shows demographic and biochemical characteristics, and dietary intake of our subjects. There was a significant difference in these data between male and female. As such, we analyzed the data separately in male and female. We examined the relationships between the genotypes/ haplotypes of the three single nucleotide polymorphisms (SNPs) (rs7069102, rs2273773, rs3818292) in the SIRT1 gene and metabolic phenotypes such as levels of fasting serum total cholesterol, LDL-cholesterol, HDL-cholesterol, triglycerides, glucose, hemoglobin A1c (HbA1c), and body mass index (BMI). These three SNPs were in linkage disequilibrium (LD) with each other $\left(\left|D^{\prime}\right|>0.87\right.$ ), and we constructed a haplotype using these three SNPs. There were three common haplotypes with frequencies of $>16 \%$, which accounted for $98 \%$ of all chromosomes in our subjects (see Additional file 1: Table S1, and Table S2).

In males, significant associations were observed between LDL-cholesterol level and all three SNPs or haplotype 2,

Table 1 Characteristics of the study subjects

\begin{tabular}{lccr}
\hline & Male & Female & P-value \\
\hline $\mathrm{n}$ & 707 & 723 & \\
Age (years) & $53.8 \pm 5.2$ & $53.0 \pm 5.1$ & 0.075 \\
BMI (kg/m ${ }^{2}$ ) & $23.5 \pm 2.9$ & $22.2 \pm 3.1$ & $<0.0001$ \\
Total Cholesterol (mg/dl) & $209.9 \pm 31.4$ & $217.7 \pm 31.4$ & $<0.0001$ \\
LDL-Cholesterol (mg/dl) & $130.4 \pm 29.8$ & $131.9 \pm 30.0$ & 0.38 \\
HDL-Cholesterol (mg/dl) & $57.0 \pm 16.3$ & $71.5 \pm 17.1$ & $<0.0001$ \\
Triglyceride (mg/dl) & $137.8 \pm 102.5$ & $86.8 \pm 45.8$ & $<0.0001^{*}$ \\
glucose (mg/dl) & $99.5 \pm 15.4$ & $92.7 \pm 10.9$ & $<0.0001^{*}$ \\
HbA1c (\%) & $5.3 \pm 0.59$ & $5.1 \pm 0.44$ & $<0.0001$ \\
Smokers (\%) & 42.5 & 8.4 & $<0.0001$ \\
Dietary intakes & & & \\
Energy (kcal/day) & $2106.5 \pm 567.0$ & $1615.5 \pm 414.9$ & $<0.0001$ \\
Total fat (\%energy) & $25.9 \pm 6.0$ & $28.2 \pm 5.7$ & $<0.0001$ \\
Carbohydrate (\%energy) & $57.1 \pm 7.8$ & $56.0 \pm 7.0$ & 0.012 \\
SFA (\%energy) & $6.5 \pm 1.8$ & $7.6 \pm 2.0$ & $<0.0001$ \\
PUFA (\%energy) & $6.7 \pm 1.7$ & $7.0 \pm 1.6$ & 0.0019 \\
n-6 PUFA (\%energy) & $5.4 \pm 1.5$ & $5.7 \pm 1.4$ & 0.0004 \\
n-3 PUFA (\%energy) & $1.5 \pm 0.49$ & $1.5 \pm 0.44$ & 0.42 \\
Alcohol (g/day) & $28.7 \pm 31.6$ & $6.5 \pm 14.6$ & $<0.0001$ \\
\hline BM body mass & & & \\
\end{tabular}

$B M I$ body mass index, HbA1c hemoglobin A1c, SFA saturated fatty acid, PUFA polyunsaturated fatty acid.

Data are expressed as mean \pm SD or percentage.

$\mathrm{P}$-values were calculated by $\mathrm{t}$-test or $\mathrm{X} 2$ test.

The subject numbers whose data for LDL-Cholesterol, HbA1c, and dietary intake available are 1430,1408 , and 1248 , respectively.

*Statistical tests for triglyceride and glucose levels were calculated on logtransformed values. 
Table 2 Relationships between genotypes/haplotypes of the SIRT1 gene and metabolic phenotypes

\begin{tabular}{|c|c|c|c|c|c|c|c|c|c|c|c|c|c|c|c|}
\hline & Genotype & & Total-Cholesterol & & LDL-Cholesterol & & HDL-Cholesterol & & Triglycerides* & & Glucose* & & $\mathrm{HbA1c}$ & & $\mathrm{BMI} * *$ \\
\hline & & $\mathrm{n}$ & $(\mathrm{mg} / \mathrm{dl})$ & $\mathrm{n}$ & $(\mathrm{mg} / \mathrm{dl})$ & $\mathrm{n}$ & $(\mathrm{mg} / \mathrm{dl})$ & $\mathrm{n}$ & $(\mathrm{mg} / \mathrm{dl})$ & $\mathrm{n}$ & $(\mathrm{mg} / \mathrm{dl})$ & $\mathrm{n}$ & $(\%)$ & $\mathrm{n}$ & $\left(\mathrm{kg} / \mathrm{m}^{2}\right)$ \\
\hline \multicolumn{16}{|l|}{ Male } \\
\hline rs7069102 & CC & 487 & $208.9 \pm 31.4$ & 482 & $129.4 \pm 29.1$ & 487 & $57.5 \pm 16.7$ & 487 & $135.2 \pm 105.4$ & 487 & $99.7 \pm 15.7$ & 484 & $5.27 \pm 0.60$ & 487 & $23.5 \pm 2.8$ \\
\hline \multirow[t]{3}{*}{ (intron 4) } & CG & 207 & $212.7 \pm 31.4$ & 203 & $133.5 \pm 31.0$ & 207 & $55.5 \pm 15.3$ & 207 & $146.0 \pm 97.3$ & 207 & $99.2 \pm 14.8$ & 206 & $5.29 \pm 0.58$ & 207 & $23.4 \pm 2.9$ \\
\hline & GG & 13 & $203.8 \pm 31.8$ & 13 & $122.7 \pm 34.3$ & 13 & $60.6 \pm 13.7$ & 13 & $100.8 \pm 55.5$ & 13 & $93.5 \pm 12.1$ & 13 & $5.33 \pm 0.61$ & 13 & $24.1 \pm 7.1$ \\
\hline & $P$ value & & 0.067 & & 0.010 & & 0.42 & & 0.017 & & 0.31 & & 0.46 & & 0.55 \\
\hline rs2273773 & $\pi$ & 315 & $212.4 \pm 33.2$ & 311 & $134.4 \pm 30.6$ & 315 & $55.3 \pm 15.7$ & 315 & $136.4 \pm 103.1$ & 315 & $99.2 \pm 14.8$ & 313 & $5.29 \pm 0.58$ & 315 & $23.4 \pm 2.9$ \\
\hline \multirow[t]{3}{*}{ (exon 5) } & TC & 294 & $208.8 \pm 29.6$ & 290 & $127.1 \pm 28.5$ & 294 & $58.1 \pm 16.4$ & 294 & $140.2 \pm 106.8$ & 294 & $99.8 \pm 16.9$ & 293 & $5.25 \pm 0.64$ & 294 & $23.5 \pm 2.9$ \\
\hline & CC & 98 & $207.8 \pm 30.4$ & 97 & $127.7 \pm 30.0$ & 98 & $58.9 \pm 17.5$ & 98 & $134.7 \pm 87.3$ & 98 & $99.5 \pm 12.2$ & 97 & $5.31 \pm 0.47$ & 98 & $23.7 \pm 2.8$ \\
\hline & $P$ value & & 0.32 & & 0.024 & & 0.054 & & 0.86 & & 0.71 & & 0.81 & & 0.40 \\
\hline rs3818292 & AA & 313 & $212.4 \pm 33.4$ & 309 & $134.3 \pm 30.6$ & 313 & $55.4 \pm 15.6$ & 313 & $136.1 \pm 103.7$ & 313 & $99.4 \pm 14.8$ & 311 & $5.29 \pm 0.59$ & 313 & $23.4 \pm 2.9$ \\
\hline \multirow[t]{3}{*}{ (intron 5) } & $A G$ & 297 & $208.0 \pm 29.4$ & 293 & $127.3 \pm 28.4$ & 297 & $57.9 \pm 16.4$ & 297 & $140.6 \pm 106.0$ & 297 & $99.7 \pm 16.9$ & 296 & $5.26 \pm 0.64$ & 297 & $23.5 \pm 3.0$ \\
\hline & GG & 97 & $207.8 \pm 30.7$ & 96 & $127.7 \pm 30.2$ & 97 & $59.1 \pm 17.8$ & 97 & $134.4 \pm 87.7$ & 97 & $99.0 \pm 12.0$ & 96 & $5.31 \pm 0.46$ & 97 & $23.7 \pm 2.7$ \\
\hline & $P$ value & & 0.27 & & 0.024 & & 0.10 & & 0.70 & & 0.75 & & 0.85 & & 0.34 \\
\hline haplotype 1 & + & 515 & $210.6 \pm 32.4$ & 510 & $131.6 \pm 30.1$ & 515 & $56.6 \pm 16.2$ & 515 & $135.7 \pm 102.2$ & 515 & $99.9 \pm 16.5$ & 513 & $5.28 \pm 0.63$ & 515 & $23.5 \pm 2.8$ \\
\hline \multirow[t]{2}{*}{$(C-T-A)$} & - & 192 & $208.1 \pm 28.6$ & 188 & $127.2 \pm 28.7$ & 192 & $58.0 \pm 16.6$ & 192 & $143.2 \pm 103.5$ & 192 & $98.2 \pm 11.6$ & 190 & $5.28 \pm 0.48$ & 192 & $23.5 \pm 3.2$ \\
\hline & $P$ value & & 0.53 & & 0.150 & & 0.24 & & 0.21 & & 0.34 & & 0.59 & & 0.86 \\
\hline haplotype 2 & + & 384 & $208.0 \pm 29.7$ & 379 & $127.4 \pm 28.8$ & 384 & $58.1 \pm 16.7$ & 384 & $139.8 \pm 102.8$ & 384 & $99.7 \pm 16.0$ & 382 & $5.27 \pm 0.60$ & 384 & $23.5 \pm 2.7$ \\
\hline \multirow[t]{2}{*}{ (C-C-G) } & - & 323 & $212.2 \pm 33.2$ & 319 & $134.1 \pm 30.5$ & 323 & $55.6 \pm 15.7$ & 323 & $135.3 \pm 102.3$ & 323 & $99.2 \pm 14.7$ & 321 & $5.29 \pm 0.58$ & 323 & $23.5 \pm 3.2$ \\
\hline & $P$ value & & 0.17 & & 0.011 & & 0.049 & & 0.47 & & 0.60 & & 0.68 & & 0.14 \\
\hline haplotype 3 & + & 213 & $212.5 \pm 31.6$ & 209 & $133.2 \pm 31.3$ & 213 & $55.5 \pm 14.9$ & 231 & $144.4 \pm 97.1$ & 213 & $99.1 \pm 14.8$ & 212 & $5.30 \pm 0.59$ & 213 & $22.4 \pm 3.2$ \\
\hline \multirow[t]{2}{*}{$(G-T-A)$} & - & 494 & $208.8 \pm 31.3$ & 489 & $129.2 \pm 29.1$ & 494 & $57.6 \pm 16.8$ & 494 & $134.9 \pm 104.8$ & 494 & $99.6 \pm 15.6$ & 491 & $5.27 \pm 0.59$ & 494 & $23.5 \pm 2.8$ \\
\hline & $P$ value & & 0.069 & & 0.055 & & 0.31 & & 0.053 & & 0.57 & & 0.37 & & 0.51 \\
\hline \multicolumn{16}{|l|}{ Female } \\
\hline rs7069102 & CC & 504 & $217.7 \pm 31.9$ & 492 & $131.7 \pm 30.9$ & 504 & $71.6 \pm 17.2$ & 504 & $85.7 \pm 43.2$ & 504 & $92.9 \pm 11.3$ & 493 & $5.14 \pm 0.45$ & 504 & $22.2 \pm 3.2$ \\
\hline \multirow[t]{3}{*}{ (intron 4) } & CG & 198 & $217.9 \pm 30.2$ & 190 & $132.0 \pm 28.0$ & 198 & $71.8 \pm 17.0$ & 198 & $87.8 \pm 50.2$ & 198 & $92.0 \pm 9.0$ & 192 & $5.16 \pm 0.40$ & 198 & $22.1 \pm 2.9$ \\
\hline & GG & 21 & $215.0 \pm 31.1$ & 20 & $134.4 \pm 26.3$ & 21 & $63.6 \pm 15.9$ & 21 & $102.8 \pm 59.6$ & 21 & $94.3 \pm 17.7$ & 20 & $5.11 \pm 0.44$ & 21 & $22.7 \pm 2.8$ \\
\hline & $P$ value & & 0.63 & & 0.82 & & 0.12 & & 0.13 & & 0.75 & & 0.20 & & 0.50 \\
\hline rs2273773 & $\pi$ & 310 & $218.6 \pm 32.0$ & 300 & $134.0 \pm 30.8$ & 310 & $70.0 \pm 16.8$ & 310 & $89.9 \pm 49.3$ & 310 & $93.3 \pm 12.9$ & 301 & $5.17 \pm 0.50$ & 310 & $22.2 \pm 3.0$ \\
\hline \multirow[t]{3}{*}{ (exon 5) } & TC & 320 & $215.6 \pm 31.0$ & 315 & $129.0 \pm 29.7$ & 320 & $72.5 \pm 17.2$ & 320 & $83.5 \pm 40.8$ & 320 & $91.8 \pm 8.9$ & 316 & $5.12 \pm 0.37$ & 320 & $22.1 \pm 3.1$ \\
\hline & CC & 93 & $221.7 \pm 30.4$ & 87 & $135.0 \pm 27.3$ & 93 & $72.8 \pm 17.7$ & 93 & $87.6 \pm 49.4$ & 93 & $93.4 \pm 10.0$ & 88 & $5.15 \pm 0.44$ & 93 & $22.6 \pm 3.4$ \\
\hline & $P$ value & & 0.23 & & 0.091 & & 0.096 & & 0.25 & & 0.14 & & 0.70 & & 0.66 \\
\hline
\end{tabular}


Table 2 Relationships between genotypes/haplotypes of the SIRT1 gene and metabolic phenotypes (Continued)

\begin{tabular}{|c|c|c|c|c|c|c|c|c|c|c|c|c|c|c|c|}
\hline rs3818292 & AA & 303 & $219.1 \pm 31.9$ & 294 & $134.4 \pm 30.4$ & 303 & $69.8 \pm 16.9$ & 303 & $90.3 \pm 49.1$ & 303 & $93.5 \pm 13.0$ & 295 & $5.17 \pm 0.50$ & 303 & $22.2 \pm 3.0$ \\
\hline \multirow[t]{3}{*}{ (intron 5) } & $A G$ & 309 & $214.9 \pm 30.8$ & 305 & $128.7 \pm 30.1$ & 309 & $72.4 \pm 17.0$ & 309 & $83.3 \pm 41.4$ & 309 & $91.7 \pm 9.1$ & 305 & $5.12 \pm 0.37$ & 309 & $22.1 \pm 3.2$ \\
\hline & GG & 111 & $221.8 \pm 30.9$ & 103 & $133.8 \pm 27.9$ & 111 & $73.4 \pm 17.8$ & 111 & $86.8 \pm 47.6$ & 111 & $93.2 \pm 9.2$ & 105 & $5.13 \pm 0.42$ & 111 & $22.5 \pm 3.30$ \\
\hline & $P$ value & & 0.11 & & 0.061 & & 0.047 & & 0.17 & & 0.090 & & 0.94 & & 0.83 \\
\hline haplotype 1 & + & 515 & $216.9 \pm 31.8$ & 504 & $131.7 \pm 31.1$ & 515 & $70.8 \pm 16.8$ & 515 & $86.6 \pm 44.2$ & 515 & $92.7 \pm 11.1$ & 505 & $5.14 \pm 0.45$ & 515 & $22.1 \pm 3.1$ \\
\hline \multirow[t]{2}{*}{$(C-T-A)$} & - & 208 & $219.7 \pm 30.1$ & 198 & $132.2 \pm 26.9$ & 208 & $73.2 \pm 17.7$ & 208 & $87.2 \pm 49.6$ & 208 & $92.7 \pm 10.5$ & 200 & $5.14 \pm 0.41$ & 208 & $22.3 \pm 3.1$ \\
\hline & $P$ value & & 0.47 & & 0.77 & & 0.045 & & 0.65 & & 0.70 & & 0.43 & & 0.88 \\
\hline haplotype 2 & + & 408 & $216.9 \pm 31.0$ & 397 & $130.1 \pm 29.4$ & 408 & $72.6 \pm 17.2$ & 408 & $84.5 \pm 43.0$ & 408 & $92.1 \pm 9.1$ & 399 & $5.13 \pm 0.38$ & 408 & $22.2 \pm 3.2$ \\
\hline \multirow[t]{2}{*}{ (C-C-G) } & - & 315 & $218.7 \pm 31.9$ & 305 & $134.1 \pm 30.6$ & 315 & $69.9 \pm 16.9$ & 315 & $89.7 \pm 49.0$ & 315 & $93.4 \pm 12.9$ & 306 & $5.16 \pm 0.50$ & 315 & $22.2 \pm 3.0$ \\
\hline & $P$ value & & 0.27 & & 0.039 & & 0.065 & & 0.16 & & 0.088 & & 0.85 & & 0.95 \\
\hline haplotype 3 & + & 216 & $217.5 \pm 30.4$ & 208 & $132.4 \pm 28.0$ & 216 & $71.0 \pm 17.1$ & 216 & $88.9 \pm 51.5$ & 216 & $92.2 \pm 10.2$ & 209 & $5.15 \pm 0.41$ & 216 & $22.2 \pm 2.9$ \\
\hline \multirow[t]{2}{*}{$(G-T-A)$} & - & 507 & $217.8 \pm 31.8$ & 494 & $131.6 \pm 30.8$ & 507 & $71.7 \pm 17.1$ & 507 & $85.8 \pm 43.1$ & 507 & $92.9 \pm 11.2$ & 496 & $5.14 \pm 0.45$ & 507 & $22.2 \pm 3.2$ \\
\hline & $P$ value & & 0.96 & & 0.67 & & 0.47 & & 0.22 & & 0.92 & & 0.29 & & 0.72 \\
\hline
\end{tabular}

Values are shown as mean \pm SD.

P-values were calculated by multiple linear regression analyses incorporating age, BMI, current smoking and alcohol intake as covariates.

*Statistical tests for glucose and TG levels were calculated on log-transformed values.

**P-values for BMI were calculated by multiple linear regression analyses incorporating age, current smoking and alcohol intake as covariates.

Statistically significant $\mathrm{P}$-values $(\mathrm{P}<0.05)$ are indicated by bold. 
Table 3 The combined effects of SIRT1 haplotype and n-6/n-3 PUFA intake ratio on serum LDL-C and HDL-C levels

\begin{tabular}{|c|c|c|c|c|c|c|c|c|}
\hline & \multicolumn{4}{|c|}{ Male } & \multicolumn{4}{|c|}{ Female } \\
\hline & \multicolumn{2}{|c|}{$\begin{array}{l}\text { Low } \mathrm{n} 6 / \mathrm{n} 3 \text { intake group } \\
(<3.79)\end{array}$} & \multicolumn{2}{|c|}{$\begin{array}{l}\text { High n6/n3 intake group } \\
(\geqq 3.79)\end{array}$} & \multicolumn{2}{|c|}{$\begin{array}{l}\text { Low } n 6 / \mathrm{n} 3 \text { intake group } \\
(<3.93)\end{array}$} & \multicolumn{2}{|c|}{$\begin{array}{l}\text { High } n 6 / n 3 \text { intake group } \\
(\geqq 3.93)\end{array}$} \\
\hline & $\bar{n}$ & (mg/dl) & $\bar{n}$ & (mg/dl) & $\bar{n}$ & (mg/dl) & $\bar{n}$ & (mg/dl) \\
\hline \multicolumn{9}{|l|}{ LDL-Cholesterol } \\
\hline Haplotype $1(+)$ & 221 & $133.5 \pm 31.1$ & 232 & $131.1 \pm 29.3$ & 215 & $133.6 \pm 30.5$ & 213 & $131.2 \pm 31.3$ \\
\hline Haplotype $1(-)$ & 88 & $127.0 \pm 29.8$ & 74 & $129.4 \pm 28.6$ & 93 & $131.3 \pm 26.2$ & 84 & $131.1 \pm 28.1$ \\
\hline P-value & & 0.13 & & 0.68 & & 0.53 & & 0.87 \\
\hline Haplotype $2(+)$ & 179 & $128.0 \pm 30.5$ & 149 & $129.4 \pm 28.6$ & 187 & $129.7 \pm 27.6$ & 163 & $130.2 \pm 30.6$ \\
\hline Haplotype $2(-)$ & 130 & $136.6 \pm 30.7$ & 157 & $131.9 \pm 29.7$ & 121 & $137.9 \pm 31.0$ & 134 & $132.4 \pm 30.1$ \\
\hline P-value & & 0.0089 & & 0.28 & & 0.0085 & & 0.68 \\
\hline Haplotype $3(+)$ & 82 & $136.8 \pm 30.2$ & 95 & $131.5 \pm 31.6$ & 82 & $131.1 \pm 25.1$ & 98 & $134.0 \pm 30.2$ \\
\hline Haplotype $3(-)$ & 227 & $129.8 \pm 30.9$ & 211 & $130.3 \pm 28.1$ & 226 & $133.6 \pm 30.6$ & 199 & $129.8 \pm 30.5$ \\
\hline P-value & & 0.033 & & 0.56 & & 0.99 & & 0.49 \\
\hline \multicolumn{9}{|l|}{ HDL-Cholesterol } \\
\hline Haplotype $1(+)$ & 223 & $55.7 \pm 16.8$ & 234 & $56.8 \pm 15.9$ & 218 & $72.1 \pm 17.2$ & 220 & $69.0 \pm 16.7$ \\
\hline Haplotype $1(-)$ & 89 & $58.3 \pm 16.7$ & 77 & $57.0 \pm 16.0$ & 96 & $76.9 \pm 17.8$ & 91 & $69.5 \pm 17.4$ \\
\hline P-value & & 0.16 & & 0.72 & & 0.022 & & 0.92 \\
\hline Haplotype $2(+)$ & 181 & $57.6 \pm 17.1$ & 152 & $57.4 \pm 16.4$ & 190 & $75.5 \pm 17.4$ & 170 & $69.4 \pm 17.1$ \\
\hline Haplotype $2(-)$ & 131 & $54.8 \pm 16.4$ & 159 & $56.4 \pm 15.4$ & 124 & $70.5 \pm 17.4$ & 141 & $68.9 \pm 16.7$ \\
\hline P-value & & 0.079 & & 0.27 & & 0.032 & & 0.83 \\
\hline Haplotype $3(+)$ & 84 & $53.8 \pm 14.9$ & 97 & $56.6 \pm 15.0$ & 84 & $71.3 \pm 17.1$ & 104 & $69.6 \pm 17.7$ \\
\hline Haplotype $3(-)$ & 228 & $57.4 \pm 17.4$ & 214 & $57.0 \pm 16.3$ & 230 & $74.4 \pm 17.6$ & 207 & $68.9 \pm 16.5$ \\
\hline P-value & & 0.14 & & 0.90 & & 0.15 & & 0.72 \\
\hline
\end{tabular}

Values are shown as mean \pm SD.

P-values were calculated by multiple linear regression analyses incorporating age, BMI, current smoking and alcohol intake as covariates

Statistically significant $P$-values $(P<0.05)$ are indicated by bold.

and between HDL-cholesterol level and haplotype 2. In females, significant associations were observed between LDL-cholesterol level and haplotype 2, and between HDLcholesterol levels and SNP (rs3818292) or haplotype 1. The other metabolic traits including serum total cholesterol, glucose, HbA1c levels, and BMI were not associated with the genotype/haplotype of the SIRT1 gene (Table 2).

The carriers of haplotype 2 had lower serum LDL-C and higher HDL-C levels than for the non-carriers of haplotype 2, although in females the relationship between HDL-C levels and haplotype 2 was not statistically significant $(P=0.065)$. These data indicate that haplotype 2 is a beneficial haplotype associated with decreased LDL-cholesterol and increased HDL-cholesterol levels.

Next, to examine whether dietary n-6 and n-3 PUFA intake modulates the association between SIRT1 haplotypes and LDL-C and/or HDL-C levels, we classified subjects into two subgroups based on the population median of dietary n-6/n-3 PUFA intake ratio (3.79 for males, 3.93 for females). Significant associations between SIRT1 haplotype 2 and LDL-C and/or HDL-C levels were observed in only the group with a low n-6/n-3 PUFA intake ratio, but were not observed in the group with a high n-6/n-3 PUFA intake ratio (Table 3). In the group with a low n-6/n-3 PUFA intake ratio, the association between haplotype 3 and LDL-C levels in male $(P=0.033)$, and that between haplotype 1 and HDL-C levels in female were also observed $(\mathrm{P}=0.022)$.

These findings indicate that the combination of genetic variations in the SIRT1 gene and dietary n-6 and/or n-3 PUFA intake influence the determination of interindividual variations of serum levels of LDL-C and HDL-C.

\section{Discussion}

Plasma levels of high LDL-C and low HDL-C are considered major determinants of susceptibility to $\mathrm{CHD}$ in the general population. The determination of plasma lipid levels is controlled by multiple pathways and influenced by complex interactions between many different genes and environmental factors such as diet intake.

In the present study, we found that the combination between the SIRT1 gene marked with haplotype 2 and low $n-6 / n-3$ PUFA intake ratio was related to beneficial effects on serum lipid profile, a decreased LDL-C and an 
increased HDL-C levels, in both males and females. Both SIRT1 and PUFAs can influence the expression of genes for nuclear receptors and transcription factors related to lipid metabolism including LXR $\alpha$, LXR $\beta$, PPAR $\alpha$, SREBP-1c [9-11,17]. Recently, it was reported that n-3 PUFAs activate AMPK/SIRT1 pathways in macrophages [19]. These data indicate the possibility that the activity or function of SIRT1 might be modified by dietary n-3 PUFAs intake. In addition, it remains possible that the activity of SIRT1 marked with haplotype 2 is more easily increased by n-3 PUFA intake without haplotype 2 . At present, we have no direct evidence that the activity of SIRT1 was influenced by dietary n-3 PUFA intake, and the SIRT1 gene marked with haplotype 2 in this study caused alterations in the activity and/or function of SIRT1 deacetylase.

Genetic variants of the SIRT1 gene have been shown to be associated with human diabetes and obesityrelated phenotypes in several previous studies [20-22], while only a few genetic association studies for the SIRT1 gene and lipid metabolism have been reported [23]. Almost all genetic variants detected in the SIRT1 gene were synonymous, only a few variants with possible functional changes were reported in the promoter region [24]. The three SNPs that we analyzed in this study are also non-functional variants. And we could not detect associations between genotype/haplotype of the SIRT1 gene and diabetes and obesity-related phenotypes including serum glucose levels, HbA1c, and BMI in this study (Table 2). However, it remains possible that the SIRT1 gene marked with haplotype 2 might exist in linkage disequilibrium with other new functional variants.

Further in vivo and in vitro studies are needed to assess the role for dietary n-3 or n-6 PUFA intake and genetic variation marked with haplotype 2 in the SIRT1 gene on changes in SIRT1 expression or function.

The lower saturated fat (meat) and higher n-3-PUFA (fish) in the Japanese diet was suggested to contribute to the lower prevalence of hypercholesterolemia and lower risk of CHD [25]. Furthermore, the beneficial SIRT1 variant marked with haplotype 2 is rather common in the Japanese population (the frequency of haplotype 2 is 0.33 ). Future studies are required to ascertain whether the combination between the SIRT1 gene marked with haplotype 2 and low n-6/n-3 PUFA intake ratio can produce beneficial effects on serum lipid profile in other populations containing young people, children, or other people with different dietary habits. Prospective cohort studies are also required to determine the interactions between genetic variations of the SIRT1 gene and dietary PUFA on serum lipid profile. It is important to determine the potential for interactions between genetic and modifiable environmental factors such as dietary nutrient intake to establish a preventive method for common diseases such as dyslipidemia and CHD.

\section{Conclusion}

We found that the genotype/haplotype of the SIRT1 gene is associated with serum LDL-C and HDL-C levels, and that the variant marked with haplotype 2 is associated with decreased LDL-cholesterol and increased HDL-cholesterol levels. In addition, the associations between the SIRT1 haplotype 2 and decreased LDL-C and/or increased HDL$C$ levels were only observed in the low $n-6 / n-3$ PUFA intake ratio group, but not in the high $n-6 / n-3$ PUFA intake ratio group. These findings indicate that the combination of genetic variations in the SIRT1 gene and dietary n-6 and/or n-3 PUFA intake can influence the determination of inter-individual variations of serum levels of LDL-C and HDL-C. An understanding of the interactions of genetic and environmental factors on the prevalence of dyslipidemia is useful for prediction and prevention of CHD, as high LDL-C and low HDL-C levels are important risk factors for CHD.

\section{Subjects and methods Subjects}

A total of 707 Japanese males and 723 females were randomly selected from participants who visited a medical center near the University of Shizuoka for routine medical check-ups. Subjects were all Japanese, and ranged from 45-65 years (mean age, 53.8 \pm 5.2 males, 53.0 \pm 5.1 females). People taking medication for dyslipidemia and/or diabetes were excluded from the study subjects. After overnight fasting, blood samples were collected from each subject. Written informed consent was obtained from all subjects and this study was approved by the Ethics Committee of the University of Shizuoka.

\section{DNA analysis}

Genomic DNA was isolated from peripheral leucocytes by the phenol extraction method. We analyzed the genotypes and haplotypes of three tag SNPs (rs7069102 [intron 4], rs2273773 [exon 5, Leu27Leu], rs3818292 [intron 5]) in the SIRT1 gene, which were selected from the HapMap database (http://hapmap.ncbi.nlm.nih.gov/index.html.en), based on their minor allele frequencies (MAF) in the Japanese population and previous reports describing the SIRT1 polymorphism [20,23]. The SIRT1 gene is localized to chromosome region 10q21.3 with 9 exons. To date, approximately 100 common SNPs $(\mathrm{MAF} \geqq 0.05)$ have been on the dbSNP database (http://www.ncbi.nlm.nih.gov/ snp?term). The majority of SNPs exist in intron or untranslated regions.

The genotypes of these three SNPs were determined for each subject using the PCR-restriction fragment length polymorphism method. The haplotypes and their frequencies were estimated by the maximum-likelihood method with an expectation-maximization-based algorithm using the SNPAlyze program (Dynacom, Tokyo, Japan). 


\section{Dietary assessment}

Dietary intake was assessed using a brief-type self-administered diet history questionnaire (BDHQ). The BDHQ was developed based on the self-administered diet history questionnaire (DHQ), which had been validated using three different standard methods for dietary assessment $[26,27]$. The BDHQ were designed to obtain dietary habits for the previous month from validating dietary intake for 58 food and beverage items which are commonly consumed in general Japan populations [26,27]. With respect to n-3 and n-6 PUFA, the majority food sources are $\alpha$-linolenic acid (vegetable oil), EPA (fish and shellfish), DHA (fish and shellfish) and linoleic acid (vegetable oil), arachidonic acid (fish, organ meats and egg), respectively. Intake of fat, carbohydrate, protein, and fatty acids was expressed as percentages of the total non-alcohol energy intake. Dietary data were available for 1248 subjects (87.3\%) in this study.

\section{Statistical analyses}

The relationships between genotypes/haplotypes of the three SNPs of the SIRT1 gene and metabolic parameters including serum lipid and glucose levels were analyzed by multiple linear regression analyses incorporating age, BMI, alcohol intake, and smoking status as covariates. Statistical analyses were performed using the JMP 9 software package (SAS Institute, Cary, NC, USA). The coefficients of linkage disequilibrium (LD) value (|D'| and $\mathrm{r}^{2}$ ) among three SNPs were calculated by using the SNPAlyze program (Dynacom, Tokyo, Japan).

\section{Additional file}

Additional file 1: Table1. The pair wise linkage disequilibrium (LD) values of |D'| (upper) and $r^{2}$ (lower). Table 2. Estimated SIRT1 haplotypes and frequencies in the Japanese population.

\section{Abbreviations}

LDL: Low density lipoprotein; HDL: High density lipoprotein; CHD: Coronary heart disease; GWAS: Genome-wide association study; SIRT1: Sirtuin 1; PPAR: Peroxisome-proliferator activated receptor; PGC-1a: Peroxisome proliferator-activated receptor gamma coactivator 1-alpha, LXR, Liver X receptor; FOXO: Forkhead box O; AMPK: AMP-activated protein kinase; SREBP: Sterol response element-binding protein; PUFA: Polyunsaturated fatty acid; SNP: Single nucleotide polymorphism; HbA1c: Hemoglobin A1c; BMI: Body mass index; LD: Linkage disequilibrium.

\section{Competing interests}

The authors declare that they have no competing interests.

\section{Authors' contributions}

$\mathrm{TI}$ and KYM managed the study and carried out the genetic analysis, drafting the manuscript. TG carried out the dietary assessment. NK collected study subjects. All authors read and approved the final manuscript.

\section{Acknowledgments}

We are grateful to the subjects for their participation in this study. We thank Prof. Satoshi Sasaki (The University of Tokyo) for his advices on dietary assessment. This study was supported by the Global COE program from the Ministry of Education, Culture, Sport, Science and Technology of Japan (MEXT).

\section{Author details}

${ }^{1}$ Laboratory of Human Genetics, School of Food and Nutritional Sciences, Graduate School of Integrated Pharmaceutical and Nutritional Sciences, University of Shizuoka, Shizuoka 422-8526, Japan. '2 Laboratory of Nutritional Physiology, School of Food and Nutritional Sciences, Graduate School of Integrated Pharmaceutical and Nutritional Sciences, University of Shizuoka, Shizuoka 422-8526, Japan. ${ }^{3}$ Department of Data Managements for Health Evaluation \& Promotion, Shizuoka Medical Center, Shizuoka 422-8033, Japan.

Received: 30 September 2012 Accepted: 5 January 2013

Published: 11 January 2013

\section{References}

1. Di Angelantonio E, Sarwar N, Perry P, Kaptoge S, Ray KK, Thompson A, Wood AM, Lewington S, Sattar N, Packard CJ, et al: Major lipids, apolipoproteins, and risk of vascular disease. JAMA 2009, 302:1993-2000

2. Weissglas-Volkov D, Pajukanta P: Genetic causes of high and low serum HDL-cholesterol. J Lipid Res 2010, 51:2032-2057.

3. Heller DA, de Faire U, Pedersen NL, Dahlen G, McClearn GE: Genetic and environmental influences on serum lipid levels in twins. $N$ Engl J Med 1993, 328:1150-1156.

4. Teslovich TM, Musunuru K, Smith AV, Edmondson AC, Stylianou IM, Koseki M, Pirruccello JP, Ripatti S, Chasman DI, Willer CJ, et al: Biological, clinical and population relevance of 95 loci for blood lipids. Nature 2010, 466:707-713.

5. Kim YJ, Go MJ, Hu C, Hong CB, Kim YK, Lee JY, Hwang JY, Oh JH, Kim DJ, Kim NH, et al: Large-scale genome-wide association studies in East Asians identify new genetic loci influencing metabolic traits. Nat Genet 2011, 43:990-995.

6. Guarente L: Sirtuins as potential targets for metabolic syndrome. Nature 2006, 444:868-874.

7. Gillum MP, Erion DM, Shulman Gl: Sirtuin-1 regulation of mammalian metabolism. Trends Mol Med 2010, 17:8-13.

8. Silva JP, Wahlestedt C: Role of Sirtuin 1 in metabolic regulation. Drug Discov Today 2010, 15:781-791.

9. Ponugoti B, Kim DH, Xiao Z, Smith Z, Miao J, Zang M, Wu SY, Chiang CM, Veenstra TD, Kemper JK: SIRT1 deacetylates and inhibits SREBP-1C activity in regulation of hepatic lipid metabolism. J Biol Chem 2010, 285:33959-33970.

10. Schug TT, Li X: Sirtuin 1 in lipid metabolism and obesity. Ann Med 2011, 43:198-211.

11. Li X, Zhang S, Blander G, Tse JG, Krieger M, Guarente L: SIRT1 deacetylates and positively regulates the nuclear receptor LXR. Mol Cell 2007, 28:91-106.

12. Kremmyda LS, Tvrzicka E, Stankova B, Zak A: Fatty acids as biocompounds: their role in human metabolism, health and disease: a review. part 2: fatty acid physiological roles and applications in human health and disease. Biomed Pap Med Fac Univ Palacky Olomouc Czech Repub 2011, 155:195-218.

13. Russo $G L$ : Dietary $n-6$ and $n-3$ polyunsaturated fatty acids: from biochemistry to clinical implications in cardiovascular prevention. Biochem Pharmacol 2009, 77:937-946.

14. Patterson E, Wall R, Fitzgerald GF, Ross RP, Stanton C: Health implications of high dietary omega-6 polyunsaturated fatty acids. J Nutr Metab 2012, 2012:ID539426.

15. Iso H, Kobayashi M, Ishihara J, Sasaki S, Okada K, Kita Y, Kokubo Y, Tsugane S: Intake of fish and n3 fatty acids and risk of coronary heart disease among Japanese: the Japan Public Health Center-Based (JPHC) Study Cohort I. Circulation 2006, 113:195-202.

16. Wall R, Ross RP, Fitzgerald GF, Stanton C: Fatty acids from fish: the antiinflammatory potential of long-chain omega-3 fatty acids. Nutr Rev 2010, 68:280-289.

17. Sampath $\mathrm{H}, \mathrm{Ntambi} J \mathrm{M}$ : Polyunsaturated fatty acid regulation of genes of lipid metabolism. Annu Rev Nutr 2005, 25:317-340.

18. Fernandes G: Progress in nutritional immunology. Immunol Res 2008 , 40:244-261. 
19. Xue $B$, Yang Z, Wang $X$, Shi H: Omega-3 polyunsaturated fatty acids antagonize macrophage inflammation via activation of AMPK/SIRT1 pathway. PLoS One 2012, 7:e45990.

20. Peeters AV, Beckers S, Verrijken A, Mertens I, Roevens P, Peeters PJ, Van Hul W, Van Gaal LF: Association of SIRT1 gene variation with visceral obesity. Hum Genet 2008, 124:431-436.

21. Zillikens MC, van Meurs JB, Rivadeneira F, Amin N, Hofman A, Oostra BA, Sijbrands EJ, Witteman JC, Pols HA, van Duijn CM, Uitterlinden AG: SIRT1 genetic variation is related to BMI and risk of obesity. Diabetes 2009, 58:2828-2834.

22. Clark SJ, Falchi M, Olsson B, Jacobson P, Cauchi S, Balkau B, Marre M, Lantieri O, Andersson JC, Jernas M, et al: Association of sirtuin 1 (SIRT1) gene SNPs and transcript expression levels with severe obesity. Obesity (Silver Spring) 2012, 20:178-185.

23. Shimoyama Y, Mitsuda Y, Tsuruta Y, Suzuki K, Hamajima N, Niwa T: SIRTUIN 1 gene polymorphisms are associated with cholesterol metabolism and coronary artery calcification in Japanese hemodialysis patients. J Ren Nutr 2012, 22:114-119.

24. Rai E, Sharma S, Kaul S, Jain K, Matharoo K, Bhanwer AS, Bamezai RN: The interactive effect of SIRT1 promoter region polymorphism on type 2 diabetes susceptibility in the North Indian population. PLoS One 2012, 7:e48621.

25. Iso H: Lifestyle and cardiovascular disease in Japan. J Atheroscler Thromb 2011, 18:83-88.

26. Sasaki S, Yanagibori R, Amano K: Self-administered diet history questionnaire developed for health education: a relative validation of the test-version by comparison with 3-day diet record in women. J Epidemiol 1998, 8:203-215.

27. Kobayashi S, Murakami K, Sasaki S, Okubo H, Hirota N, Notsu A, Fukui M, Date C: Comparison of relative validity of food group intakes estimated by comprehensive and brief-type self-administered diet history questionnaires against $16 \mathrm{~d}$ dietary records in Japanese adults. Public Health Nutr 2011, 14:1200-1211.

\section{doi:10.1186/1476-511X-12-4}

Cite this article as: Inamori et al:: The combined effects of genetic variation in the SIRT1 gene and dietary intake of $n-3$ and $n-6$ polyunsaturated fatty acids on serum LDL-C and HDL-C levels: a population based study. Lipids in Health and Disease 2013 12:4.

\section{Submit your next manuscript to BioMed Central and take full advantage of:}

- Convenient online submission

- Thorough peer review

- No space constraints or color figure charges

- Immediate publication on acceptance

- Inclusion in PubMed, CAS, Scopus and Google Scholar

- Research which is freely available for redistribution 